\title{
Behandlung von Augentumoren mit Protonenstrahlen
}

\section{Martin Jermann}

Dipl. Phys. ETH, Stabschef und Vizedirektor Paul Scherrer Institut PSI, Villigen

Der Autor ist Referent an den «Trendtagen Gesundheit Luzern» (siehe Kasten nächste Seite), wo er zu dem Thema «Die Protonentherapie - von der Idee bis zum Produkt der Spitzenmedizin» sprechen wird.

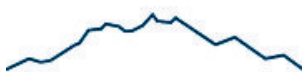

Trendtage Gesundheit Luzern
* OPTIS ist in Europa eine Pionieranlage für die Therapie von Augentumoren mit Protonenstrahlen. Inzwischen sind in Europa nach dem PSI-Vorbild sechs weitere derartige Einrichtungen in Betrieb.

Korrespondenz:

Paul Scherrer Institut Dipl. Phys. Martin Jermann CH-5234 Villigen

martin.jermann@psi.ch

\section{Einleitung}

Bald 30 Jahre ist es her, dass Physiker am Schweizerischen Institut für Nuklearforschung SIN (eines der beiden Vorgängerinstitute des Paul Scherrer Instituts, PSI) den Entscheid trafen, die für Grundlagenforschung in der Physik gebaute Protonen-Beschleunigeranlage für die Bestrahlung von Augentumoren mit Protonenstrahlen zu nutzen. Die Idee dazu stammte aus den USA: Am Massachusetts General Hospital in Boston/ Harvard Cyclotron war kurz zuvor erfolgreich ein ähnliches Projekt lanciert worden. In weniger als 2 Jahren wurde am PSI ein entsprechender Bestrahlungsplatz aufgebaut. In enger Zusammenarbeit mit dem Hôpital Ophtalmique Jules Gonin der Universität Lausanne wurden im März 1984 am PSI erstmals in Europa Patientinnen und Patienten, die an Tumoren des Auges litten, mit Protonenstrahlen behandelt. Auch heute, nach mehr als 25 Jahren, ist diese Zusammenarbeit äusserst erfolgreich: Über 200 Augentumor-Patienten aus der Schweiz und aus ganz Europa profitieren jährlich von dieser äusserst schonenden und effizienten Krebstherapie am PSI.

Inzwischen hat sich die Protonentherapie weit über die Behandlung von Augentumoren hinaus in der Strahlentherapie etablieren können. Für bestimmte, sehr schwierig zu behandelnde Tumoren ist sie heute die Therapiemethode der ersten Wahl. Für viele Universitätsspitäler im In- und Ausland ist die Protonentherapie deshalb zu einem Prestigeobjekt geworden. Sie gehört in die Kategorie der Spitzenmedizin

\section{Aderhautmelanom: \\ jährlich 50 bis 60 neue Fälle}

Der häufigste primäre Tumor im Auge ist das sogenannte Aderhautmelanom. Es entwickelt sich aus entarteten Pigmentzellen in der Aderhaut des Auges. In Europa treten auf eine Million Einwohner jährlich ca. 6-10 Fälle auf, am häifigsten betroffen sind Personen im Alter zwischen 50 und 70 Jahren. In der Schweiz werden etwa 50-60 derartige Tumorerkrankungen pro Jahr diagnostiziert. Die Bestrahlung der Augentumoren mit Protonenstrahlen ist sehr schonend; dies hat mit den besonderen physikalischen Eigenschaften der Protonen zu tun.

Zur Behandlung der Augentumoren mit Protonenstrahlen müssen die Protonen auf relativ hohe Energien beschleunigt werden. Dies geschieht in einem sogenannten Zyklotron. Zuerst werden die Protonen

\section{Traitement des tumeurs oculaires par protonthérapie}

Cela fait presque 30 ans que les physiciens de l'Institut suisse de recherche nucléaire (un des deux instituts précurseurs de I'Institut Paul Scherrer (PSI) ont décidé d'utiliser l'accélérateur à protons, destiné à la recherche fondamentale en physique, pour le traitement des tumeurs oculaires. L'idée venait des EtatsUnis et plus particulièrement du Cyclotron du Massachusetts General Hospital à Boston/Harvard, où un projet du même type venait d'être lancé. En moins de deux ans, le PSI a créé un poste d'irradiation. Grâce à une étroite collaboration avec I'Hôpital Ophtalmique Jules Gonin de I'Université de Lausanne, le PSI a pu traiter pour la première fois en Europe, en mars 1984, des patients atteints de tumeurs oculaires par protonthérapie. Aujourd'hui encore et plus de 25 ans après, cette collaboration demeure fructueuse: plus de $\mathbf{2 0 0}$ patients de Suisse et d'Europe bénéficient chaque année de ce traitement contre le cancer très efficace et ultra précis.

Depuis lors, la protonthérapie, bien établie au sein de la radiothérapie, a fait ses preuves contre d'autres types de tumeurs. Pour les tumeurs spécifiques et difficiles à traiter, elle représente aujourd'hui un traitement de premier choix. De nombreux hôpitaux universitaires en Suisse et à l'étranger la considèrent comme prestigieuse et l'incluent dans la médecine de pointe.

aus Wasserstoff gewonnen, dabei wird Wasserstoffgas auf sehr hohe Temperaturen erhitzt und ionisiert. Die Kerne der Wasserstoffatome, die Protonen, werden anschliessend im Zyklotron auf Geschwindigkeiten von über 100000 Kilometern pro Sekunde beschleunigt. Das Protonen-Zyklotron ist ein Kreisbeschleuniger, in dem Protonen aus dem Zentrum der Anlage 
spiralförmig nach aussen fliegen und dabei auf definierte hohe Geschwindigkeiten beschleunigt werden. Der Protonenstrahl, der einen Protonenfluss von mehreren Milliarden Protonen pro Sekunde aufweist, wird, mit Magneten gelenkt, durch ein weitgehend luftfreies (evakuiertes) Rohr zur Bestrahlungseinrichtung - der OPTIS-Anlage - geführt.

\section{Schonung des Sehnervs}

Die beschleunigten Protonen werden beim Eintritt ins Auge abgebremst, sie verlieren Energie. Durch Variation der Einfallsenergie kann die Eindringtiefe ins Auge genau gesteuert werden. Erst wenn die Protonen in definierter Tiefe auf dem Augenhintergrund, das heisst im Tumor, stoppen, deponieren sie den Grossteil der Strahlendosis und zerstören damit die Tumorzellen. Strukturen hinter dem Auge, wie z. B. der Sehnerv, werden vor unerwünschter Strahlenbelastung geschont. Die Präzision der Augenbestrahlung wird zusätzlich durch eine seitliche Formung des Protonenstrahls erhöht: Der einfallende Strahl wird vor dem Eintritt ins Auge durch eine Blende geleitet, welche die Umrisse des Tumors aus der Sicht des einfallenden Protonenstrahls abbildet und die Protonen ausserhalb der Tumorumrisse abschirmt. Der Tumor wird so mit den einfallenden Protonen bis zur vorausberechneten

\begin{abstract}
«Herausforderung Innovation»
6. Trendtage Gesundheit Luzern am 17. und 18. März

Innovation ist Erneuerung. Ob erforscht, entwickelt oder zufällig entdeckt, wir erwarten von Innovationen immer eine Verbesserung bisheriger Zustände. Gerade der medizinische Sektor ist geprägt durch innovative Entwicklungen. Sie sind zugleich Fundus und Triebfeder unseres qualitativ hochstehenden Gesundheitswesens, haben aber oft auch erhebliche Mehrkosten zur Folge. Was steckt aber genau hinter einer Innovation? Welche Innovationen benötigt die Medizin dringend? Wie finanzieren wir diese? Wie werden innovative Errungenschaften unter DRGs zum Standard? Diese und weitere Fragen stehen im Mittelpunkt der kommenden Tagung.

Die Trendtage Gesundheit Luzern thematisieren Perspektiven und Trends im Gesundheitswesen. Sie bieten eine kompetente Plattform für Wissenstransfer und Meinungsbildung an und wollen damit Entscheidungsgrundlagen für ein effizientes sowie gesellschaftlich und finanziell tragbares Gesundheitswesen in der Schweiz bereitstellen. Darüber hinaus sollen sie eine jährliche Standortbestimmung zu ausgewählten Fragen über das nationale und gegebenenfalls auch das internationale Gesundheitswesen ermöglichen. Der Kongress richtet sich an Entscheidungsträger aller wichtigen Bereiche des Gesundheitssektors. Erwartet werden rund 400 Besucher und Besucherinnen.

Der Schweizerische Ärzteverlag EMH ist Medienpartner der 6. Trendtage Gesundheit Luzern.
\end{abstract}

Datum: Mittwoch, 17. März, und Donnerstag, 18. März 2010

Ort: KKL Luzern, Luzerner Saal

Programm/Informationen: Details zum Kongress sind auf der Webseite www. trendtage-gesundheit.ch zu finden. Weitere Auskünfte unter der Telefonnummer 0413183797.

Anmeldung: Anmeldungen werden entgegengenommen unter www.trendtagegesundheit.ch

Anmeldeschluss ist der 15. März 2010. klinisch festgelegten Dosis präzise bestrahlt. Das umliegende (gesunde) Gewebe des Auges, insbesondere auch die Eintrittsstelle des Sehnervs ins Auge und die Makula (Bereich des schärfsten Sehens), werden dadurch optimal vor Strahlung geschützt, soweit die Lage des Tumors und seine Nähe zu empfindlichen Strukturen dies erlauben.

Die Behandlungen der Augentumoren werden an vier aufeinanderfolgenden Tagen durchgeführt, wobei in der Regel für jede «Bestrahlungs-Sitzung» eine Dosis von 15 Gray in das Tumorvolumen abgegeben wird. Damit die hohe Präzision der Bestrahlung erzielt werden kann, sind umfangreiche Vorkehrungen und Vorbereitungen notwendig. Die Positionierung des Patientenauges muss millimetergenau mit den geplanten und vorausberechneten Daten der Bestrahlung übereinstimmen. Die Bestrahlung selbst dauert etwa eine Minute, aber die Vorbereitungen und Einstellungen nehmen für jeden Patienten täglich 15 bis 20 Minuten in Anspruch.

\section{4 erste Krebspatienten am PSI erfolgreich behandelt}

Anfang der 80er Jahre wurden erste Ideen entwickelt, am $72 \mathrm{MeV}$-Injektor der Protonenbeschleunigeranlage, die am damaligen Schweizerischen Institut für Nuklearforschung (SIN) für die Grundlagenforschung in der Physik gebaut worden war, eine Bestrahlungseinrichtung für Augentumoren zu errichten. Dank dieser Pionierarbeiten, die zusammen mit dem Hôpital Ophtalmique Jules Gonin der Universität Lausanne durchgeführt wurden, konnten im März 1984 erste Krebspatienten am PSI erfolgreich mit Protonenstrahlen behandelt werden. Bis Ende 2009 wurden an dieser Bestrahlungsanlage (OPTIS) 5300 Krebspatienten therapiert - die höchste Anzahl mit Protonenstrahlen behandelter Augentumoren weltweit -, wobei die Erfolgsrate für die Behandlung der Primärtumoren im Durchschnitt bei 98 Prozent liegt. Damit nimmt das PSI in der Protonentherapie international einen Spitzenplatz ein.

Im Jahr 2009 wurden über 220 Patientinnen und Patienten mit bösartigen Tumoren im Auge am PSI behandelt. Dazu kamen 120 Therapien mit der PSIProtonengantry bei Patientinnen und Patienten mit tiefliegenden Tumoren im Körper; etwa ein Drittel der Patienten sind Jugendliche und Kleinkinder, die ganz besonders von dieser schonenden und effizienten Krebstherapie mit Protonenstrahlen profitieren können.

Das PSI steht weltweit an der Spitze der technologischen Entwicklung. Zurzeit baut es seine Anlage weiter aus. In Zukunft soll die am PSI entwickelte hochpräzise Protonentherapie-Technik für weitere schwer zu behandelnde, bösartige Tumoren (z. B. Lungen- und Brustkarzinome) verfügbar sein. Dafür entwickelt das PSI ein neues Bestrahlungsgerät, Gantry 2, mit dem Tumorbewegungen während der Bestrahlung berücksichtigt und kompensiert werden können, so dass die hohe Präzision der Bestrahlung erhalten bleibt. 